Mots. Les langages du politique

\title{
Glasnost et perestroika. Les pérégrinations de deux russismes dans la presse française
}

Sarah Leroy

\section{(2) OpenEdition \\ Journals}

Édition électronique

URL : https://journals.openedition.org/mots/17253

DOI : $10.4000 /$ mots. 17253

ISSN : 1960-6001

Éditeur

ENS Éditions

Édition imprimée

Date de publication : 1 novembre 2006

Pagination : 65-78

ISBN : 978-2-84788-099-1

ISSN : 0243-6450

Référence électronique

Sarah Leroy, "Glasnost et perestroika. Les pérégrinations de deux russismes dans la presse

française », Mots. Les langages du politique [En ligne], 82 | 2006, mis en ligne le 01 novembre 2008, consulté le 23 avril 2022. URL : http://journals.openedition.org/mots/17253 ; DOI : https://doi.org/ $10.4000 /$ mots. 17253

\section{(c) ENS Éditions}




\title{
Méthodes
}

\author{
Sarah Leroy
}

\section{Glasnost et perestroïka. Les pérégrinations de deux russismes dans la presse française}

La présence de mots d'origine russe dans le lexique français est assez discrète : l'étude exhaustive d’Éva Buchi (à paraître) ne relève que 225 russismes intégrés au français. Cela se traduit par une très faible représentation dans la lexicographie courante : seuls environ 80 emprunts au russe sont présents dans un dictionnaire d'usage comme le Petit Robert électronique (1996).

Parmi les derniers arrivés figurent deux russismes qui ont été très en vogue dans la presse française entre la fin des années quatre-vingt et le début des années quatre-vingt-dix, glasnost et perestroïka. Fermant la liste des emprunts désignant des institutions soviétiques ${ }^{1}$, ces deux mots ont été introduits et véhiculés par la presse française ${ }^{2}$ tout comme, vraisemblablement, par celle des autres pays européens et occidentaux à la même époque ${ }^{3}$. Leur irruption soudaine dans le discours de la presse française, avec un pic entre 1989 et 1991 (le graphique ci-dessous présente la fréquence des deux termes dans les titres de presse présents dans la base Europresse ${ }^{4}$ de 1986 à 2005) n’a pas, comme on

1. É. Buchi (à paraître) indique que les $27,5 \%$ des russismes du français fournis par la période de la «Russie bolchevique, puis soviétique (1917-1990) » relèvent massivement du vocabulaire politique et institutionnel, à la différence de ceux des périodes précédentes.

2. Les premières mentions sont en effet signalées dans la presse ; le Petit Robert considère ainsi que glasnost apparaît le 16 décembre 1986 dans Le Nouvel Observateur. La Base d'observation et de recherche des néologismes (Bornéo) de l’Atilf (www.atilf.fr) le signale cependant, ainsi que perestroïka, dès le 21 février 1986.

3. É. Buchi (à paraître) signale leur arrivée en italien, catalan, espagnol et portugais entre 1986 et 1989 et, un peu plus tardivement (1991), pour des raisons historiques évidentes, en roumain.

4. www.europresse.com. Cette base de données permet (après abonnement) de consulter le texte intégral d'une dizaine de quotidiens et hebdomadaires français, avec une ancienneté variable selon les titres.

Université Paris X-Nanterre, Modèles, dynamiques, corpus, sarah.leroy@u-paris1o.fr 
aurait pu le croire, été suivie d'une disparition tout aussi soudaine : après une stabilisation en 1993, leur présence se maintient désormais à un niveau faible, mais régulier.

\section{Fréquence Europresse}

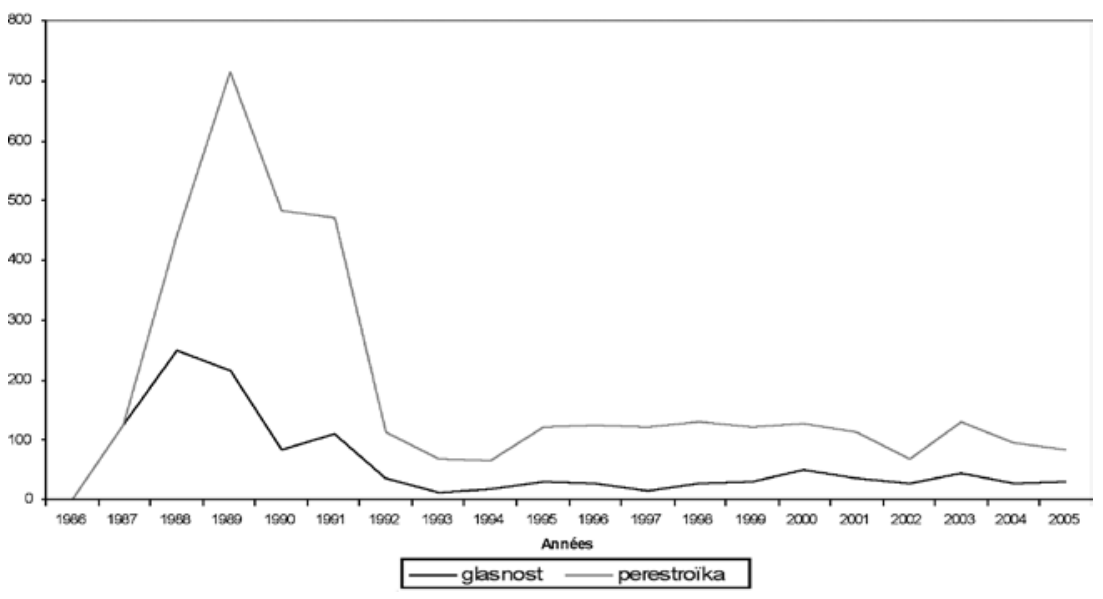

\section{Emprunts, xénismes, pérégrinismes?}

"L'intégration sémantique des russismes au français est assez peu avancée » (Buchi, à paraître), au point qu'on peut se demander, pour les deux termes qui nous occupent, s'ils sont réellement intégrés à la langue ou s'ils demeurent des éléments définitivement étrangers au sein des discours, voire de la langue française. En effet, même si leur lexicographisation ${ }^{5}$, dans les dictionnaires les plus courants, est effective, on ne peut pas pour autant considérer qu'ils sont totalement intégrés au lexique français au point de - comme tant d'emprunts plus anciens - ne plus être sentis comme provenant d'une autre langue. Pour tenter d'observer, avec glasnost et perestroïka, un processus d'emprunt et non le simple usage de termes empruntés, on doit faire appel aux notions de xénisme et de pérégrinisme.

En effet, si le terme générique emprunt désigne « une unité ou un trait linguistique qui existait précédemment dans un parler $B$ (dit langue source) et que A ne possédait pas, [utilisé et intégré dans] un parler A» (Jean Dubois et al., 1994, p. 177), un xénisme est « une unité lexicale constituée par un mot d'une

5. La lexicographisation, c'est-à-dire la présence dans les dictionnaires de langue, n'est pas la lexicalisation, l'entrée d'un mot dans un dictionnaire, tout comme sa sortie, dépendant de paramètres linguistiques, mais aussi extralinguistiques. On peut cependant, tout en gardant ces restrictions à l'esprit, considérer la lexicographisation comme un critère "objectif » et, bien que non dénué d'arbitraire, relativement fiable, d'« entrée en langue» - donc de lexicalisation - d'un emprunt. 
langue étrangère et désignant une réalité propre à la culture des locuteurs de cette langue » (Dubois et al., 1994, p. 512). Entre ces deux extrêmes se trouve un moyen terme, le pérégrinisme (Dubois et al., ibid., p. 512). Dans ce continuum, « le xénisme est un mot étranger, mentionné avec référence au code linguistique d'origine et aux réalités étrangères, [le] pérégrinisme renvoie encore à la réalité étrangère, mais la connaissance de son sens est supposée partagée par l'interlocuteur », et l'emprunt marque le dernier stade, celui de l'intégration achevée.

Si les distinctions que marquent ces trois termes ne sont ni nettes ni étanches, elles présentent l'avantage de mettre la question de l'emprunt en relation avec les usages discursifs, en soulignant que l'intégration d'un terme étranger dépend étroitement d'aspects tels que le temps, la fréquence et la perception de l'extranéité marquée en discours par divers procédés ${ }^{6}$, dont la glose. Selon Dubois et al. (ibid., p. 512), le passage du xénisme au pérégrinisme se marque en effet par le fait que ce dernier relève d'une «utilisation occasionnelle mais dépourvue de marques linguistiques». Certains critères sémantiques (Buchi, à paraître) sont des indicateurs d'intégration. Ainsi, si le sémantisme du mot n'est plus en rapport avec la civilisation d'origine, on pourra parler d'emprunt achevé; si seules des acceptions secondaires s'en sont détachées, il faudra plutôt considérer qu'on a affaire à un pérégrinisme. Par ailleurs, l'existence de formations secondaires, de dérivés lexicalisés ou occasionnels indique généralement une intégration linguistique avancée, sans pour autant qu'on puisse en faire la marque de l'emprunt établi.

Parmi les russismes répertoriés dans le Petit Robert électronique, seule une dizaine peuvent sans hésitation être considérés comme des emprunts définitivement intégrés : si la rubrique étymologique mentionne l'emprunt au russe, la définition elle-même soit ne renvoie pas du tout à la réalité russe (mammouth est ainsi défini comme un « grand éléphant fossile du quaternaire à l'épaisse toison et aux longues défenses recourbées vers le haut»), soit présente une acception «secondaire » du point de vue diachronique, mais première du point de vue de l'usage, qui est coupée de cette réalité russe (oukase a un sens 1 , "Hist. », d' "édit promulgué par le tsar», et un sens 2, "Fig.», de "décision arbitraire, ordre impératif»). Ainsi, la grande majorité des russismes relève soit du xénisme, pour les mots qui demeurent clairement étrangers et pour lesquels la définition renvoie au monde et à la civilisation russes (touloupe, par exemple, est dit désigner une « veste en peau de mouton portée par les paysans russes »), soit du pérégrinisme pour ceux (rouble, samovar, moujik, goulag...) qui, tout en renvoyant à une réalité russe ou soviétique, sont suffisamment intégrés pour pouvoir être employés sans explication particulière et développent parfois une acception secondaire éloignée du contexte original (on peut ainsi

6. Voir Guérin, dans ce même volume. 
qualifier, par métaphore, un endroit particulièrement pénible de goulag). Ces trois « états » étant en fait trois paliers, il est souvent délicat de trancher entre une catégorie et une autre : doit-on parler de xénisme ou de pérégrinisme pour isba, de pérégrinismes ou d'emprunts pour blinis, spoutnik?

C'est la question que l'on va se poser pour glasnost et perestroïka, dont le second est justement choisi par Dubois et al. (ibid., p. 512) pour illustrer les différents stades de l'emprunt: "On peut dire que perestroïka a connu les deux stades du xénisme et puis du pérégrinisme sans passer à l'emprunt; le processus dénoté n'a pas perdu sa référence à l'URSS. » Du point de vue lexicographique, du moins pour ce qui concerne le Petit Robert électronique, glasnost et perestroïka se situent nettement du côté des emprunts non intégrés, xénismes ou au moins pérégrinismes ; le cadre russe ou soviétique est en effet explicitement mentionné dans les deux cas : outre l'indication de l'origine linguistique dans la rubrique étymologique, la définition s'ouvre sur «En URSS» pour perestroïka et «En Union soviétique » pour glasnost. Pour aller plus loin, les outils de l'analyste de discours vont venir compléter ceux du lexicologue et permettre d'observer plus finement la relative intégration de ces mots dans le cadre de la presse française.

En effet, l'emprunt de glasnost et perestroïka n'est pas seulement un transfert linguistique mais «le passage d'un objet de discours [...] d'une culture à une autre, avec les altérations et adaptations que cela implique » (Jean-Marc Sarale dans Catherine Détrie et al., 2001, p. 369). Tous deux constituent finalement des «formules», au sens de Pierre Fiala (dans Patrick Charaudeau et Dominique Maingueneau, 2002, p. 274-275) : renvoyant à des notions «ayant joué sur le plan idéologique un rôle fondateur et actif dans une situation historique », leur « contenu référentiel n'est pas un concept stable ». L'arrivée de ces russismes sur le « marché linguistique » est bien un phénomène qui relève d'une moderne langue de bois : ces deux mots sont des étendards, les symboles d'un changement politique, et sont utilisés comme tels par le discours des médias. En effet, il n'y a pas dans ce cas de contact linguistique direct entre deux communautés, mais un contact indirect qui passe par les médias et, en fait, ne les quitte guère. Ces nouvelles réalités, évènements et processus historiques pour lesquels aucun terme n'était disponible en français (ni, vraisemblablement, en russe, où glasnost et perestroïka semblent être des néologismes de sens), font l'objet « de controverses, de définitions contradictoires, d'affrontements polémiques » (Fiala, dans Charaudeau et Maingueneau, ibid., p. 274) qui transparaissent jusque dans leurs emplois les plus neutres. Ces mots, finalement, paraissent moins empruntés en tant que mots de la langue qu'en tant que mots du discours et c'est pourquoi les commentaires, les reformulations et les paraphrases mettent particulièrement au jour les enjeux politiques et discursifs qui s'y nouent. Nous nous limiterons ici à l'étude de la glose (voir Steuckardt et Niklas-Salminen, 2003), c'est-à-dire aux commentaires faits par les 
locuteurs eux-mêmes sur les mots qu'ils emploient, aux segments qui « posent conventionnellement que tel mot soit entendu hic et nunc dans telle acception " (Julia, 2001, p. 102), à l'aide d'un outil de liaison ou pas. C'est en observant ainsi l'usage qu'on mesurera à la fois l'intégration des russismes dans le discours de la presse et les contournements dont leur sens fait l'objet.

\section{Comment glose-t-on les emprunts?}

Les occurrences de glasnost et perestroïka dans la presse française ont été observées sur un important corpus constitué essentiellement des données de la base Europresse et, en complément, de celles d'un cédérom Europresse ${ }^{7}$, ainsi qu'à l'aide du moteur de recherche Glossanet ${ }^{8}$. Ces deux outils ont permis d'explorer huit titres majeurs de la presse française (Le Point, Le Monde diplomatique, Libération, Le Monde, Les Échos, L'Express, Le Figaro, L'Humanités) sur une période allant de 1980 à 2005 mais couvrant essentiellement les quinze dernières années. Ce qui frappe cependant, sur les quelque 5000 mentions de l'un ou l'autre terme, c'est la proportion étonnamment faible, même dans les premiers temps, d'occurrences glosées : on peut estimer qu'elles représentent, dans l'ensemble, $5 \%$ des cas. La proportion des occurrences glosées diffère cependant, selon les périodes mais aussi selon les termes : glasnost, moins fréquent que perestroïka, semble plus glosé. À titre d'exemple, nous présentons les données d'un hebdomadaire, Le Monde diplomatique, où se trouvent, de 1986 à 2005, 470 occurrences de perestroïka, dont seulement 17 sont accompagnées d'une glose, et 139 occurrences de glasnost dont 22 glosées.

\section{Gloses de perestroikka et de glasnost dans Le Monde diplomatique}

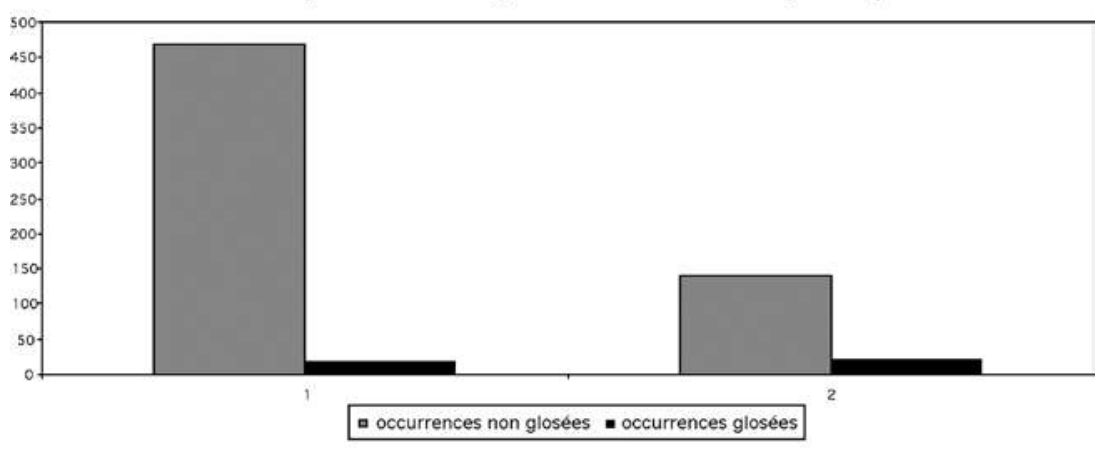

7. Pour Le Monde diplomatique.

8. glossa.fltr.ucl.ac.be. Ce moteur de recherche permet de repérer des attestations de mots ou de structures syntaxiques sur l'édition quotidienne en ligne d'une quarantaine de quotidiens francophones.

9. On compte également une occurrence tirée de Paris-Match. 
Au sein du total d'environ 250 occurrences glosées que nous observons, on relève différents types de glose, diverses manières de mettre en relation glasnost et/ou perestroïka avec un segment glosant, traduction, commentaire ou développement. Les possibilités ne sont cependant pas infinies; comme le remarque A. Niklas-Salminen (2003, p. 71), « le geste méta-énonciatif d'explicitation du sens d'une unité lexicale empruntée [...] est beaucoup plus simple que celui d'une unité lexicale ordinaire ». Trois types de glose sont représentés parmi nos occurrences : outre la simple juxtaposition, par apposition (virgules, parenthèses, tirets, deux points), structure de loin la plus fréquente, la glose peut s'accompagner d'un marqueur ${ }^{10}$, que ce soit un commentaire métalinguistique intégré à une structure appositive ou présenté de façon plus libre ou, plus rarement, un connecteur. Dans tous les cas, le mot glosé change de statut sémiotique : du fait même de la présence de la glose, il acquiert une connotation autonymique ${ }^{11}$ qui peut être soulignée typographiquement ou non.

\section{Les gloses apposées}

L'apposition, sous ses diverses formes, juxtapose au russisme un équivalent français. Le russisme est alors généralement en usage et l'équivalent français en mention (1), bien que les appariements de deux termes en usage (2), voire en mention si le contexte s'y prête, ne soient pas exceptionnels :

(1) Cette visite démontrera la « glasnost » (transparence) ${ }^{12}$ de l'Union soviétique dans les affaires internationales, a estimé M. Piadychev, après avoir rappelé que Moscou a récemment ouvert la station radar de Krasnoyarsk, en Sibérie, à des membres du Congrès américain. (Le Monde, 3 octobre 1987)

(2) L'époque était à la glasnost (la transparence) et les Russes, pleins d'espérance, se tournaient pour la première fois vers la télévision et la radio d'État dans l'espoir d'y reconnaître un peu d'eux-mêmes. (L'Express, 31 aout 2000)

Lorsque, plus rarement, c'est le terme français qui est donné en premier et que le russisme est en position de glose, le fonctionnement sémiotique reste le même : le premier terme est, sauf exception, en usage, le second (le russisme) étant majoritairement en mention (3) :

(3) Que disent ces «histoires» du Moscou d'aujourd'hui? Que la «transparence»

10. Voir Steuckardt et Niklas-Salminen, 2005.

11. C'est-à-dire que son énonciation se double d'une représentation de cette énonciation, qu'il se trouve à la fois en mention et en usage. Selon Josette Rey-Debove (1971, p. 92), il s'agit d'un «téléscopage de l'emploi ordinaire des signes et de leur emploi autonymique, par lequel l'encodeur emploie pour parler du monde (et non des signes) une séquence rapportée, de telle sorte qu'il emploie et qu'il cite tout à la fois. [...] La séquence ne se signifie pas elle-même, mais se connote par un effet de sens".

12. Dans les exemples, c'est nous qui soulignons, sauf mention contraire. 
(glasnost) médiatique déborde largement, désormais, la dénonciation des déficiences de l'économie et de la société, qu'elle atteint le fonctionnement des institutions, du parti, de la justice. Qu'au moment où, en Occident, beaucoup d'observateurs se demandent encore si cette «transparence » n'est pas tout simplement une «opération de charme » destinée aux pays de l'Ouest, en URSS, deux années consécutives de «reconstruction » (perestroïka) gorbatchévienne soulèvent des questions d'un tout autre genre. (Le Monde Diplomatique, mai 1987)

Dans cet ordre, plus inattendu que l'ordre inverse, la glose n'intervient pas pour aider la compréhension, mais pour donner un équivalent «local », ce qui indique que le mot français, même s'il s'agit d'une traduction exacte du mot russe, n'est qu'une approximation qui ne suffit pas à en rendre tout le sens.

\section{Les gloses introduites par un marqueur métalinguistique}

Lorsque les deux termes sont reliés par un commentaire métalinguistique, celuici peut proposer une équivalence ou introduire un jugement. On range dans la première catégorie les formules qui renvoient à la traduction (4), voire à une sorte de définition (5):

(4) À première vue, il pourrait y avoir deux raisons péremptoires pour ne pas lire l'article publié dans la dernière livraison de la revue trimestrielle Politique étrangère [en italique dans le texte] sous le titre "L'Occident peut-il soutenir la perestroïka ?». La première est que la perestroïka (signifiant: reconstruction) est, politiquement, remise en cause, de façon tragique, par la répression dans les pays baltes. La deuxième raison est qu'économiquement la situation paraît encore plus désespérée. (Le Monde, 12 décembre 1991)

(5) Selon Alexeï Simonov, le directeur de l'organisation non gouvernementale (ONG) Fonds pour la défense de la glasnost (terme faisant référence à la relative libéralisation de l'information en URSS à l'époque Gorbatchev), «les autorités continuent à purger le secteur de l'information de tout ce qu'elles considèrent comme représentant une opposition ». (Le Monde, 2 avril 2002)

Le deuxième groupe de gloses métalinguistiques est constitué par celles qui renvoient à la qualification, et à la façon de nommer l'objet (6 et 7) :

(6) En Occident, on a écrit des livres sur la langue soviétique, sur son contenu idéologique qui permet de manipuler la parole. On comprend bien pourquoi, vu l'importance accordée à l'époque de la perestroïka à cette arme puissante que l'on appelle la glasnost. (Le Monde, 3 mars 1990)

(7) Le putsch du mois d'août à Moscou et son échec n'ont pas mis fin au désordre soviétique, mais ils ont relancé de plus belle la liberté d'expression et de déballage (ne parlons plus de glasnost, un terme tout aussi dépassé que celui de perestroïka). (Le Monde, 5 décembre 1991) 
On change alors d'orientation: les gloses d'équivalence articulent un russisme à un équivalent français, tandis que celles qui introduisent un commentaire qualifiant présentent la notion en français avant de donner le russisme. L'exemple 7, remettant en cause l'appellation elle-même et son adéquation à la réalité désignée, avance déjà une discussion sur le sens, que l'on retrouvera dans les exemples 27 et 28 .

\section{Les gloses introduites par un connecteur}

Enfin, même si l'emploi des connecteurs est plutôt rare dans la glose des emprunts (Niklas-Salminen, 2003, p. 67 et 60-61), on trouve quelques occurrences de glose introduite par ou (plutôt) ou c'est-à-dire, voire à savoir (8 à 11) :

(8) La glasnost, ou transparence, arrive enfin, parce que les choses se sont accélérées brusquement avec l'insurrection armée, dans le Chiapas, le $1^{\mathrm{er}}$ janvier de cette année, d'une poignée de guérilleros mayas de l'Armée zapatiste de libération nationale (EZLN), sous la houlette d'un leader masqué, au discours étonnamment modéré et démocrate, qui se fait appeler le « sous-commandant Marcos ». (L'Express, 18 aout 1994)

(9) Quelle révolution, ou plutôt quelle perestroïka, les nouveaux dirigeants imaginent-ils imposer au PCC? (Le Figaro, 25 avril 2003)

(10) La participation des travailleurs et la "glasnost» (transparence), c'est-à-dire la libre discussion des problèmes économiques et sociaux, constituent, selon lui, la meilleure garantie du succès d'une réforme qui s'impose, reconnaît-il, « lentement, difficilement, douloureusement ». (Le Monde, 10 novembre 1987)

(11) L'autre face de ce document, pourtant, c'est d'être la première manifestation formelle de ce que l'on appelle ailleurs "glasnost », à savoir une volonté de réforme et d'ouverture. (Le Monde, 8 septembre 1987)

On note également que ces trois principales structures peuvent se superposer les unes aux autres, des marqueurs métalinguistiques ou des connecteurs se trouver juxtaposés au terme glosé (4 et 8), des connecteurs succéder à une glose parenthétique (10).

\section{De la traduction à l'intégration}

Si l'on s'en tient aux occurrences présentées jusqu'ici, l'intégration de glasnost et perestroïka en français semble en effet bien peu avancée; sans demeurer des xénismes absolus (la faible proportion d'occurrences glosées indique au contraire que les réalités soviétiques auxquelles renvoient ces termes sont supposées connues des lecteurs), ils ne semblent pas avoir dépassé le stade du 
pérégrinisme, mais bien n'être employés que pour désigner ces réalités étrangères : renvoyant à une culture autre, ils restent étrangers à la langue. L'intégration n'est cependant pas nulle, et l'analyse du contenu de la glose, de la nature de l'équivalence ou du commentaire qu'elle apporte, permet d'en déceler quelques indices et d'observer la direction dans laquelle s'effectue cette intégration.

\section{Des traductions plus ou moins fidèles}

La plupart du temps, les gloses introduisent simplement une traduction: un mot français est présenté comme l'équivalent du mot russe. II s'agit en fait d'une traduction de l'acception empruntée plutôt que du mot russe lui-même : les traductions les plus fréquemment données sont en effet transparence, pour glasnost (12), et restructuration ou, en seconde position, reconstruction, pour perestroïka (12 et 13), trois équivalents qui ne reprennent pas la totalité du sens des termes russes ${ }^{13}$, mais seulement leur acception dans le contexte politique qui a conduit à leur emprunt :

(12) Il est tentant de dresser un parallèle avec l'explosion de la centrale atomique de Tchernobyl, quelques semaines seulement après la glasnost et la perestroïka (transparence et restructuration) initiées naguère par le président Gorbatchev. Avec les conséquences que l'on sait. (Le Figaro, 3 mai 2003)

(13) En quelques mois, l'histoire s'est accélérée. Difficile de dire ce que constituent, dans la matérialité sociale, trois années de «perestroïka » (reconstruction, restructuration), depuis le plénum d'avril 1985 jusqu'à la conférence nationale de juin 1988, en passant par le vingt-septième congrès du parti et la cascade de lois organisant les réformes économiques. (Le Monde Diplomatique, juillet 1988)

Ces équivalents fonctionnent comme des traductions «officielles», vraisemblablement proposées par les agences de presse: tous les titres, quelle que soit leur orientation politique, les utilisent majoritairement. Pour autant, ils ne s'interdisent pas de prendre des distances avec ces traductions, soit en y apportant des commentaires ou des précisions, soit en en imaginant d'autres. Parmi les commentaires autour de la traduction canonique, on rencontre des procédés de mise à distance : c'est la fonction de l'adjectif fameux, accompagnant

13. C'est surtout le cas pour glasnost qui, formé sur glasny, « rendu public » (Petit Robert électronique) pourrait également se traduire par publicité, diffusion; Serguei Sakhno (2005, p. 68) note d'ailleurs le principe d'étymologie populaire, enclenchée par la traduction courante transparence, selon lequel les «francophones interprètent [glasnost] comme étant en rapport avec fr. glace "miroir" et angl. glass "verre" » alors que « son sens en russe est plutôt « "possibilité de tout dire à haute voix" ». Perestroïka, signifiant reconstruction (d'une maison, de la société) ou transformation (de la personnalité) et utilisé dans des métaphores marxistes comme reconstruction de l'édifice étatique (Sakhno, 2005, p. 203), se voit spécialisé dans le domaine politique et institutionnel par la traduction restructuration. 
aussi bien le mot français (14) que le mot russe (15), qui met à distance la traduction « autorisée» :

(14) Les deux nouveautés du "gorbatchévisme» concernent d'abord l'étendue de la réforme qui doit toucher l'ensemble de l'économie et non quelques secteurs ou entreprises expérimentaux, et ensuite la " glasnost», la fameuse «transparence», qui doit permettre au peuple de s'exprimer, de critiquer les récalcitrants et d'ouvrir la voie à l'initiative individuelle dans tous les domaines. (Le Monde, 16 décembre 1987)

(15) La même logique se retrouve dans l'escalade incessante des mots d'ordre en deux ans: de l'« accélération » annoncée au début, l'on est vite passé à la "restructuration » - la fameuse perestroïka -, puis à la « révolution » et à la «réforme radicale». Le tout sur fond de "transparence» (la non moins fameuse glasnost) et de « démocratie ». (Le Monde, 3 novembre 1987)

Un certain nombre de commentaires, visant à préciser ou nuancer la traduction canonique, vont dans le même sens et font apparaître que l'équivalent proposé n'est pas une traduction stricte et exacte (16 et 17) :

(16) La libération d'Andreï Sakharov (décembre 1986) et de nombreux dissidents ouvre la voie à la glasnost (transparence dans la presse) et à la perestroïka. (Le Monde, 5 décembre 1991)

(17) La perestroïka a un complément: la glasnost, expression généralement traduite par «transparence », qui exprime le fait de rendre quelque chose public. (Le Monde, 22 mai 1999)

Enfin, il arrive que le russisme soit utilisé pour gloser un terme d'une autre langue (18), ce qui indique qu'il est suffisamment intégré au français pour introduire un xénisme absolu, donc qu'il a lui-même dépassé ce stade :

(18) Après dix-neuf ans d'isolement et de léthargie communiste, voici toutefois le pays lancé dans le « chintanakan mai », la perestroïka. (L'Express, 26 mai 1994)

Les traductions canoniques sont par ailleurs concurrencées par plusieurs autres, qui tendent à établir non pas le sens des mots russes, mais celui des russismes désormais français : il s'agit donc moins d'une traduction stricte que de définitions du sens d'unités nouvellement intégrées en français. Tout en restant relatifs à la réalité étrangère, ils intègrent en partie la vision occidentale en tout cas française - de cette réalité. Perestroïka se voit ainsi glosé par des traductions plus approximatives (réforme, refonte) accompagnées parfois de commentaires et de précisions (19 et 20):

(19) À partir de 1988, en effet, Ceausescu devient encombrant pour Moscou et Washington. Pour Gorbatchev, le dictateur est un frein à sa politique de réforme, la perestroïka. (Le Figaro, 25 février 2004) 
(20) Cette réforme économique, qui constitue le cœur de la refonte (perestroïka) dans laquelle M. Mikhaïl Gorbatchev a engagé l'Union soviétique, est la sixième depuis 1957. (Le Monde Diplomatique, janvier 1988)

Pour glasnost, la diversité est plus grande encore : publicité, franchise, ouverture, liberté d'expression, information, souci de vérité (21 à 23)... On s'éloigne alors nettement de la traduction plus ou moins exacte du russe pour glisser vers le commentaire du russisme :

(21) De la politique de glasnost, de franchise, à laquelle le numéro un soviétique s'était engagé, voilà bien le trait le plus saisissant. (Le Monde Diplomatique, janvier 1987)

(22) Si Mikhaïl Gorbatchev n’a pas oublié les auteurs soviétiques dans son appel à la glasnost (l'ouverture), la littérature tchèque, pour sa part, attend encore - peutêtre en vain - qu'on lui rende une liberté comparable. (Le Monde Diplomatique, mars 1987)

(23) Mais c'est dans le domaine de la liberté d'expression (glasnost) que triomphe la perestroïka. (Le Monde Diplomatique, septembre 1989)

\section{De la traduction au commentaire}

Une certaine intégration apparaît dès lors que les gloses s’éloignent de la traduction, même approximative, pour s'orienter vers le commentaire. Certaines gloses, proposant des interprétations sensiblement nouvelles, mettent ainsi en lumière une progressive assimilation sémantique (24 à 26) :

(24) La perestroïka - l'espoir de changements - et la glasnost - la possibilité de le formuler - expliquent que les questions nationales remontent aujourd'hui à la surface, le vide législatif et l'inexpérience de la démocratie font qu'elles dégénèrent en conflits, parfois sanglants. (Le Monde Diplomatique, juin 1989)

(25) C'est pourquoi aussi, en Union soviétique, la modernisation de l'économie, la "perestroïka », s'accompagne de la transparence, de la « glasnost»; l'une peut difficilement aller sans l'autre. (Le Monde Diplomatique, novembre 1988)

(26) Or c'est cette règle d'or du système stalinien qui se trouve aujourd'hui mise en cause par la « perestroïka », et surtout par son corollaire, la " glasnost», la tentative de démocratisation du débat politique. (Le Monde, 26 mars 1988)

Le russisme flanqué de ces commentaires tend à s'éloigner de son domaine conceptuel initial; la distance augmente encore lorsque la réalité culturelle d'origine est mise en doute, niée ou contredite, lorsque l'équivalence proposée va à l'encontre des équivalences habituelles (27 et 28) ou, juxtaposée à un terme de la guerre froide, se joue d'une vision « politiquement correcte » qui pose justement la fin de cette guerre froide (29) : 
(27) Comment ne pas se défendre? Comment ne pas haïr cette destruction inattendue de tout ce qui était habituel et paisible, cette destruction appelée "perestroïka et glasnost »? (Le Monde, 26 juillet 1989)

(28) Trois écologistes venus de Moscou ont indiqué que "glasnost ne veut pas dire liberté de parole » en URSS. Tous, pourtant, demandaient à leurs homologues occidentaux de soutenir la perestroïka mise en route par M. Mikhaïl Gorbatchev. (Le Monde, 11 avril 1989)

(29) Présentée en compétition officielle au dernier festival de Cannes, cette « Oreille » faisait partie du lot venu avec le dégel, pardon, la perestroïka. (Les Échos, 4 janvier 1991)

Un stade supplémentaire, et décisif, est franchi lorsque la glose indique que l'emploi des russismes se fait sans aucune relation au contexte russe, mais qu'ils désignent des réalités chinoise (30), européenne (31), franco-française (32). Ces gloses constituent alors un commentaire interprétatif, fortement orienté (en particulier par la coprésence de nomenklatura), qui souligne, sinon la perte du lien avec la réalité russe, du moins l'apparition d'acceptions secondaires assez différentes et éloignées :

(30) Comme à Moscou, la "perestroïka » chinoise (la politique de libéralisation de l'économie engagée en 1978 par Deng Xiaoping autour de la «modernisation » et de l'« ouverture ») alimente, à juste titre, la fierté des dirigeants du pays. (Les Échos, 6 novembre 2002)

(31) Michel Badoc, professeur à HEC et conseiller à la SOFRES, préconise dans Banques et Assurances, réussir l'Europe [en italique dans le texte], une "perestroïka », réforme en profondeur « des structures, des méthodes et des mentalités ». (Les Échos, 26 mars 1991)

(32) La nomenklatura française [...] rêve d'une perestroïka, c'est-à-dire de demiréformes, de demi-mesures qui infléchissent le système tout en préservant les privilèges. (Paris-Match, 5 juin 1997)

Ces timides marques d'intégration sont relativement récentes ; un pas supplémentaire semble avoir été franchi encore plus récemment, par certaines occurrences métaphoriques - non glosées - dans lesquelles les russismes, repris en tant que noms d'évènements, voire noms de processus, s'inscrivent dans une structure binominale $\mathrm{N}_{1}$ de $\mathrm{N} 2$, particulièrement propice à la métaphore et au déplacement conceptuel (33 et 34). On est alors bien loin du contexte russe, et la possibilité de métaphore montre qu'un certain degré d'intégration a été atteint, dans un certain type de discours de presse tout au moins :

(33) Le micro de Canal + était une tentative d'ouverture, dans un univers hippique auquel on reproche souvent son opacité. La "glasnost du turf» initiée par Canal + vient de prendre du plomb dans l'aile [...]. (Libération, 19 février 2005) 
(34) Le départ de Jacques Chirac provoquerait en tout cas des changements. Qui embrassera les vaches s'il est mis au régime ? Sarkozy et Villepin n'ont pas le look. Remarquons en outre que la glasnost sanitaire n'apporte pas toutes les réponses. On a tous les bulletins de santé de George W. Bush et on ne sait quand même pas ce qu'il a. (Libération, 10 septembre 2005)

Peut-on finalement considérer que glasnost et perestroïka sont intégrés dans le lexique français, devenus de vrais emprunts ? L'ensemble du parcours présenté jusqu'ici semble indiquer le contraire. Ces russismes ont été relativement intégrés, de façon très rapide, jusqu'à un stade moyen, celui du pérégrinisme : leur intégration morphologique, par exemple, n'est pas nulle, puisque perestroïka a donné lieu à quelques formations secondaires (Castroïka, Béréstroïka ou Paristroïka), selon le procédé morphologique original (faisant de -stroïka un suffixe) de l' « affixation sécrétive » (Fradin, 2003, p. 212). L'analyse des occurrences en discours, et tout particulièrement des gloses qui y sont relatives, vient ici confirmer et appuyer les données lexicologiques. L'utilisation massive, dans la presse française, de l'emprunt pour rendre compte des évènements qui ont marqué la fin de l'URSS montre bien que ces réalités étaient alors profondément étrangères, en quelque sorte insaisissables, et ont été décrites avec des mots tout aussi étrangers, ce qui a entraîné une vision schématique, voire stéréotypée, de la réalité. Le caractère systématique, et vraisemblablement concerté au sein des acteurs de la presse, des traductions transparence et reconstruction, masquant le maintien de l'opacité de la signification des mots russes et de la réalité des évènements qui se déroulent alors en URSS, va en ce sens, et rares sont les textes qui mettent en lumière, par des gloses introduisant un commentaire ou une mise en garde, l'incompréhension que masque, finalement, l'usage (voire l'abus) de glasnost et perestroïka dans les médias. La faible intégration sémantique des russismes confirme que les mots ont finalement masqué, en les caractérisant de façon rudimentaire, les faits, lesquels, comme les emprunts les désignant, sont restés lointains et étrangers.

\section{Références}

BUCHI E., à paraître, Bolchevik, mazout, toundra et les autres. Dictionnaire des emprunts au russe dans les langues romanes. Inventaire. Histoire. Intégration, Paris, CNRS.

CHARAUDEAU P. et MAINGUENEAU D. éd., 2002, Dictionnaire de l'analyse du discours, Paris, Le Seuil.

DÉTRIE C., SIBLOT P. et VÉRINE B. éd., 2001, Termes et concepts pour l'analyse du discours. Une approche praxématique, Paris, Champion. 
DUBOIS J., GUESPIN L., GIACOMO M., MARCELLESI C., MARCELLESI J.-B., MEVEL J., 1994, Dictionnaire de linguistique et des sciences du langage, Paris, Larousse.

FRADIN B., 2003, Nouvelles approches en morphologie, Paris, PUF.

JULIA C., 2001, Fixer le sens? La sémantique spontanée des gloses de spécification du sens, Paris, Presses de la Sorbonne Nouvelle.

NIKLAS-SALMINEN A., 2003, "Les emprunts et leur glose», SteuckardtA. et NiklasSalminen A. éd. (voir infra), p. 57-72.

REY-DEBOVE J., 1971, «Notes sur une interprétation autonymique de la littérarité : le mode du “comme je dis" ", Littérature, $\mathrm{n}^{\circ}$ 4, p. 90-95.

SAKHNOS., 2005, 100 racines essentielles du russe: Découvrir les trésors des mots, Paris, Ellipses.

STEUCKARDT A. et NIKLAS-SALMINEN A. éd., 2003, Le mot et sa glose, Aix-en-Provence, Publications de l'Université de Provence.

- éd., 2005, Les marqueurs de glose, Aix-en-Provence, Publications de l'Université de Provence. 